\title{
Effects of the absorbent types on changes in benzo[a]pyrene and volatile compounds in sesame oil
}

Sang Mi Lee ${ }^{1 \dagger}$, Ji-Yoon Jeong ${ }^{2 \dagger}$, Joon-Goo Lee ${ }^{3}$ and Young-Suk Kim²*

\begin{abstract}
The effects of different absorbent types on changes in benzo[a]pyrene (BaP) and volatiles in sesame oil during filtration processes were investigated using gas chromatography-mass spectrometry analysis. The BaP content was greatly reduced after filtration using powdered activated carbons (activated carbon made from peat: PP, activated carbon made from coconut shell: PC, activated carbon made from wood: PW) in comparison to granular activated carbons (activated carbon made from coconut shell: GC, activated carbon made from wood: GW). The BaP content in sesame oil was also considerably reduced when filtrated with a PW-acid clay mixture in comparison to PW-kaolin and PW-celite mixtures. Most volatile compounds were also greatly reduced after filtration using powdered activated carbons (PP, PC, and PW) in comparison to granular activated carbons (GC and GW). These results might be due to the relatively ionic structure on the surface and internal surface area of absorbent pores.
\end{abstract}

Keywords: Absorbents, Activated carbon, Benzo[a]pyrene, Sesame oil, Volatile compounds

\section{Introduction}

Sesame oil obtained from roasted sesame seeds is widely used as a seasoning ingredient, salad oil, and cooking oil in various Asian countries due to its distinctive flavor and pleasant taste [1]. Sesame oil also has a long shelf life, high oxidative stability, and beneficial health functions such as anti-inflammation and antimutagenic activity [2, 3].

The manufacturing processes for sesame oil involve the selection, cleaning, roasting, grinding, cooking, and pressing of sesame seeds, and additional processes such as refining and filtering $[4,5]$. The roasting process is a particularly important step in sesame oil production as its flavor characteristics and quality could be influenced by the conditions used, such as temperature and time [6]. The roasting process at a high temperature $\left(220-260{ }^{\circ} \mathrm{C}\right)$

\footnotetext{
*Correspondence: yskim10@ewha.ac.kr

† Sang Mi Lee and Ji-Yoon Jeong contributed equally to this work

${ }^{2}$ Department of Food Science and Engineering, Ewha Womans

University, Seoul 03760, Republic of Korea

Full list of author information is available at the end of the article
}

can lead to the generation of benzo[a]pyrene $(\mathrm{BaP})$, which can be a key harmful compound due to its carcinogenicity and mutagenicity, as well as other various volatile compounds $[7,8]$.

Recently, several studies have been conducted to reduce $\mathrm{BaP}$ in sesame oil using absorbents during the refining and filtration processes $[9,10]$. Various adsorbents, such as activated carbons, clay materials, agricultural wastes, and siliceous materials, were applied to remove the hazardous compounds [11]. Choi et al. [10] reported that activated carbons were more efficient in removing the $\mathrm{BaP}$ in sesame oil compared with other adsorbents such as kaolin, perlite, acid clay, and celite. Activated carbons had the highest absorption capacity regarding polycyclic aromatic hydrocarbons (PAHs) such as BaP, due to their well-developed porous properties and wide range of polarity derived from functional groups on the surface of activated carbons [12].

The manufacturing process can also change the flavor characteristics of sesame oil, and it has been used to improve the organoleptic quality of sesame oil. In 
particular, the overall flavor of sesame oil considerably depends on the roasting process, and the effects of the roasting process on these flavor characteristics of sesame oil have been studied [6]. Nakamura et al. [13] identified 221 compounds including pyrazines, pyrroles, pyridines, furans, and sulfur-containing compounds in the oil produced from roasted sesame seeds. Among these, pyrazines (e.g., acetyl pyrazine and alkyl pyrazines) and sulfur-containing compounds (e.g., 2-mercaptoacetaldehyde and hydrogen sulfide) seemed to significantly affect the flavor characteristics of the oil produced from roasted sesame seeds [13]. In addition, volatile compounds such as pyrazines, pyrroles, furans, and sulfur-containing compounds can be produced through various reactions such as Strecker degradation, the Maillard reaction, and lipid oxidation in sesame oil [14, 15]. Lee et al. [16] suggested that various pyrazines can be considered important contributors to the organoleptic properties of roasted sesame oil.

While many studies have investigated the effects of various adsorbents on $\mathrm{BaP}$ reduction in foods, no previous study has investigated the effects of various adsorbents such as activated carbons on the changes in volatile profiles during the filtration process. Accordingly, the present study aimed to determine the effects of various adsorbents on changes in $\mathrm{BaP}$ and volatile compounds in sesame oil during the filtration process.

\section{Materials and methods}

\section{Materials and chemicals}

Sesame oil was obtained from Chung-Yang food company (Anseong, Gyeonggi-do, Korea). Five adsorbents of activated carbons used in the present study were as follows: powdered type activated carbon made from peat (PP), granular type activated carbon made from coconut shell (GC), powdered type activated carbon made from coconut shell (PC), granular type activated carbon made from wood $(\mathrm{GW})$, powdered type activated carbon made from wood (PW). PC, GW, and PW were obtained from Shin Kwang chemical industry (Yangsan, Gyeongnam, Korea). Two types (PP, GC) of activated carbons were purchased from O-Sung Company (Seoul, Korea) and Samchully Activated Carbon Company (Geumsan, Chungcheongnam-do, Korea), respectively. In addition, celite and kaolin were purchased from Sigma-Aldrich (St. Louis, MO), and acid clay was obtained from Duk-San Science (Seoul, Korea).

Dichloromethane, $\mathrm{n}$-Hexane, and $\mathrm{N}$-paraffin oil were obtained from J.T. Baker (Philipsburg, NJ). $p$-Cymene was purchased from Sigma-Aldrich (Saint Louis, MO). $\mathrm{N}, \mathrm{N}$-Dimethylformamide was purchased from Avantor Performance Materials (Central Valley, PA). benzo[a] pyrene $(\mathrm{BaP})$ and benzo $[a]$ pyrene- $d_{12}\left(\mathrm{BaP}-d_{12}\right)(>99 \%)$ were purchased from Supelco (Bellefonte, PA, USA).

\section{Sample preparation}

Sesame oil was stored at room temperature in a dark place before analysis of volatiles. Each BaP $(5 \mu \mathrm{g} / \mathrm{kg})$ was added in sesame oil, and then all samples were stored in the dark for $18 \mathrm{~h}$ at room temperature. After that, each sesame oil sample (100 g) containing BaP were filtrated with $0.5 \%(\mathrm{w} / \mathrm{w})$ of activated carbons such as PP, GC, PC, GW, and PW for analyses of benzo[a]pyrene and volatile compounds.

Other adsorbents such as celite, kaolin, and acid clay were used to investigate the effects of the mixtures of activated carbon and other adsorbents on the changes of $\mathrm{BaP}$ and volatile profiles. All sesame oil samples were stirred at $600 \mathrm{rpm}$ for $30 \mathrm{~min}$ at $70{ }^{\circ} \mathrm{C}$, and then treated with the mixtures of activated carbon and other adsorbents at the ratio of 1:1 $(w / w)$. All experiments were conducted in triplicate.

\section{Ananlysis of benzo[a]pyrene in Sesame oil}

Sesame oil sample $(10 \mathrm{~g})$ and $200 \mu \mathrm{L}$ of BaP-d $\mathrm{d}_{12}[200 \mu \mathrm{g} / \mathrm{L}$ $(\mathrm{w} / \mathrm{v})$ in dichloromethane] as an internal standard were added thoroughly mixed in $\mathrm{n}$-hexane solution $(100 \mathrm{~mL})$. The mixtures were extracted twice with $25 \mathrm{~mL}$ of $\mathrm{N}, \mathrm{N}$ dimethylformamide ( $N, N$-DMF): water $(9: 1, \mathrm{v} / \mathrm{v})$ solution. After that, the extract [N,N-DMF: water $(9: 1, \mathrm{v} / \mathrm{v})$ phase] was added in $100 \mathrm{~mL}$ of $1 \%$ sodium sulfate solution and $50 \mathrm{~mL}$ of $\mathrm{n}$-hexane solution. The extracts were centrifuged at $4{ }^{\circ} \mathrm{C}$ and $3500 \mathrm{rpm}$ for $10 \mathrm{~min}$ (UNION 32R PLUS; Hanil Science Industrial, Incheon, Korea). After separation, the hexane phase was washed two times with $50 \mathrm{~mL}$ water. The organic extract was treated with sodium sulfate to eliminate the remaining moisture, and then concentrated to $2 \mathrm{~mL}$ using a rotary evaporator (SB-1000; EYELA Co, Tokyo, Japan). The extracts $(2 \mathrm{~mL})$ were purified using bonded phase silica SPE cartridges (Sep-Pak silica, $500 \mathrm{mg}$ stationary phase, $6 \mathrm{~mL}$ capacity; Agilent Technologies, Palo Alto, CA). Cartridges were conditioned with $10 \mathrm{~mL}$ of dichloromethane and $20 \mathrm{~mL}$ of n-hexane, and then the concentrated samples were loaded. Then the cartridges were washed with $5 \mathrm{~mL}$ of $\mathrm{n}$-hexane before $\mathrm{BaP}$ was eluted with $15 \mathrm{~mL}$ of $\mathrm{n}$-hexane: dichloromethane $(3: 1, \mathrm{v} / \mathrm{v})$. The elution of $\mathrm{BaP}$ was concentrated using a gentle flow of nitrogen gas, and then the residue was dissolved with $200 \mu \mathrm{L}$ of dichloromethane.

The analysis of $\mathrm{BaP}$ compounds was carried out using a $6890 \mathrm{~N}$ gas chromatograph connected to a 5975 mass spectrometer (Agilent Technologies, Santa Clara, CA, USA). A DB-5MS capillary column (30 m length $\times 0.25 \mathrm{~mm}$ I.D., $0.25 \mu \mathrm{m}$ film thickness, J\&W Scientific, Folsom, CA, USA) was used with helium gas 
as carrier gas at a constant flow rate of $1.5 \mathrm{~mL} \min -1$. The injection volume was $1 \mu \mathrm{L}$ in splitless mode at $320{ }^{\circ} \mathrm{C}$ injector temperature. The oven temperature was initially at $100{ }^{\circ} \mathrm{C}$ and held for $10 \mathrm{~min}$, increased to $280{ }^{\circ} \mathrm{C}$ at $60^{\circ} \mathrm{C} / \mathrm{min}$, and then raised to $310^{\circ} \mathrm{C}$ at $60{ }^{\circ} \mathrm{C} /$ min and held for $10 \mathrm{~min}$. The transfer line temperature was $280{ }^{\circ} \mathrm{C}$, the mass spectrometer scanned from $\mathrm{m} / \mathrm{z}$ 35 to 500 in electron impact (EI) mode at $70 \mathrm{eV}$. All samples were analyzed in triplicate.

For identification and quantification of BaP, selected ion monitoring (SIM) detection by GC-MS was used. $\mathrm{BaP}$ were identified using authentic standard compounds by matching their retention times and mass spectral data. BaP was detected at $m / z 252,253$, and 250, whereas BaP- $\mathrm{d}_{12}$ was detected at $m / z 264,265$, and 260. For quantification of BaP, the $m / z 252$ and 264 were used for $\mathrm{BaP}$ and $\mathrm{BaP}-\mathrm{d}_{12}$, respectively. The quantitative analysis was carried out on the base of calibration curve. The calibration curve was applied by various concentrations of $\mathrm{BaP}$ solutions in the range of $0.05-10 \mu \mathrm{g} / \mathrm{L}$ in dichloromethane $(w / v)$, added with a fixed amount $(10 \mu \mathrm{g} / \mathrm{L})$ of $\mathrm{BaP}_{-} \mathrm{d}_{12}$ as an internal standard. The calibration curve was determined using the ratios of the area of $\mathrm{BaP}$ versus the area of $\mathrm{BaP}-\mathrm{d}_{12}$. The linearity value of $R^{2}=0.9992$ were obtained.

\section{Analysis of volatile compounds in Sesame oil}

$20 \mathrm{~mL}$ of sesame oil and $0.3 \mathrm{~mL}$ of $p$-cymene [1 mg/ $\mathrm{mL}(\mathrm{w} / \mathrm{v})$ in paraffin oil] as an internal standard were put into $50 \mathrm{~mL}$ screw vial with a screw cap and kept at $30{ }^{\circ} \mathrm{C}$ for $1 \mathrm{~h}$. After the equilibrium, volatiles in headspace were adsorbed onto SPME fiber coated with $50-30 \mu \mathrm{m}$ divinylbenzene/carboxen/polydimethylsiloxane (DVB/CAR/PDMS) purchased from Supelco (Bellefonte, PA, USA) at $30{ }^{\circ} \mathrm{C}$ for $10 \mathrm{~min}$. Then, desorption was performed at $250{ }^{\circ} \mathrm{C}$ for $5 \mathrm{~min}$ in a GC injector. All experiments were performed in triplicate.

Analysis of volatile compounds in sesame oil was performed by $6890 \mathrm{~N} \mathrm{GC}$ system coupled to 5975 mass selective detector equipped with a DB-5MS column (30 $\mathrm{m} \times 0.25 \mathrm{~mm}, 0.25 \mu \mathrm{m}$ film thickness, J \& W Scientific). The oven temperature was initially held at $40{ }^{\circ} \mathrm{C}$ for $6 \mathrm{~min}$, and then increased to $140{ }^{\circ} \mathrm{C}$ at $3{ }^{\circ} \mathrm{C} / \mathrm{min}$, and then to $200{ }^{\circ} \mathrm{C}$ at $60^{\circ} \mathrm{C} / \mathrm{min}$. Other GC-MS conditions were based on the previous method of Lee et al. (2016).

Volatile compounds in sesame oil were tentatively identified using Wiley $7 \mathrm{n}$ mass spectral database and linear retention indices (RI) values. Volatile compounds were quantified by comparing their peak areas with that of $p$-cymene as internal standard.

\section{Statistical analysis}

Analysis of variance (ANOVA) was performed using SPSS (version 12.0, Chicago, IL, U.S.A.) by Duncan's multi range test to evaluate significant differences $(\mathrm{p}<0.05)$ in samples.

\section{Results and discussion \\ Effects of different types of activated carbons on BaP reduction}

Sesame oil samples with additional BaP $(5 \mu \mathrm{g} / \mathrm{kg})$ were filtrated with $0.5 \%(\mathrm{w} / \mathrm{w})$ activated carbons, such as PP, GC, PC, GW, and PW. Figure 1 shows the BaP contents in sesame oil samples filtrated by different types of activated carbons. PW significantly reduced the BaP level in sesame oil compared with that of the control $(4.55 \mu \mathrm{g} / \mathrm{kg})$ to $1.58 \mu \mathrm{g} / \mathrm{kg}$, followed by PP $(1.68 \mu \mathrm{g} / \mathrm{kg})$, PC $(2.08 \mu \mathrm{g} /$ $\mathrm{kg}), \mathrm{GW}(2.29 \mu \mathrm{g} / \mathrm{kg})$, and GC $(2.47 \mu \mathrm{g} / \mathrm{kg})(p<0.05)$.

Among the activated carbons, PW and PP were adsorbents that effectively reduced the $\mathrm{BaP}$ level of the sesame oil compared with PC, GW, and GC, indicating that polarity of activated carbon could affect $\mathrm{BaP}$ absorption in sesame oil. The internal surface areas of mesopores with $1.7-10.0 \mathrm{~nm}$ diameter in activated carbons ranged from 21.14 to $406.75 \mathrm{~m} 2 / \mathrm{g}$. PW had the largest internal surface area $(406.75 \mathrm{~m} 2 / \mathrm{g})$ of mesopores with 1.7 $10.0 \mathrm{~nm}$ diameter in activated carbons, followed by GW $\left(305.70 \mathrm{~m}^{2} / \mathrm{g}\right)$, PC $\left(203.50 \mathrm{~m}^{2} / \mathrm{g}\right)$, PP $\left(137.15 \mathrm{~m}^{2} / \mathrm{g}\right)$, and GC $\left(21.14 \mathrm{~m}^{2} / \mathrm{g}\right)$. Sesame oil sample filtrated with GC showed the smallest reduction ratio of $\mathrm{BaP}$ in sesame oil possibly due to the smallest value of internal surface area of mesopores with 1.7-10.0 nm diameter. In the case of

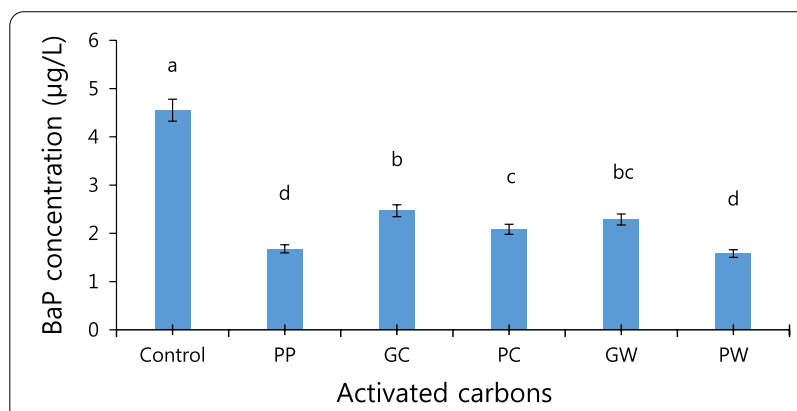

Fig. 1 Effects of different types of activated carbons on the reduction of benzo[a]pyrene in sesame oil. The symbols of the samples are as follows: Control, sesame oil sample with no treatment; PP, sesame oil sample filtrated with powdered activated carbon made from peat; GC, sesame oil sample filtrated with granular activated carbon made from coconut shell; PC, sesame oil sample filtrated with powdered activated carbon made from coconut shell; GW, sesame oil sample filtrated with granular activated carbon made from wood; PW, sesame oil sample filtrated with powdered activated carbon made from wood. Data are expressed as mean $\pm \operatorname{SEM}(n=3)$. The presence of different letters $(a-d)$ indicates a statistical difference at $P<0.05$ between bars 
$\mathrm{PW}$, it showed the highest reduction ratio of $\mathrm{BaP}$ in sesame oil samples due to the highest internal surface area of mesopores with 1.7-10.0 nm diameter. On the other hands, PP and PW had the higher carbon/oxygen ratio of internal surface on particles compared to that of other granular types of activated carbons (GC and GW) (data not shown). Accordingly, the reduction of $\mathrm{BaP}$ in sesame oil samples could be affected by the internal surface area of mesopores with 1.7-10.0 nm diameter and carbon/ oxygen ratio of internal surface on particles including internal surface of pores in activated carbons. Therefore, these activated carbons, particularly, powdered activated carbons, could be used to reduce $\mathrm{BaP}$ content in sesame oil products, minimizing their quality change.

\section{Effects of combining activated carbon and other adsorbents on $\mathrm{BaP}$ reduction}

Figure 2 shows the contents of $\mathrm{BaP}$ in sesame oil filtrated with a mixture (at a 1:1 ratio) of PW and other adsorbents, such as acid clay, kaolin, and celite, at a 600-rpm agitation speed. PW was the most effective adsorbents for the reduction of $\mathrm{BaP}$ levels in sesame oil $(1.02 \mu \mathrm{g} /$ $\mathrm{kg})$, followed by a mixture acid of PW and clay $(1.26 \mu \mathrm{g} /$ $\mathrm{kg})$, a mixture acid of PW and kaolin $(2.04 \mu \mathrm{g} / \mathrm{kg})$, and a mixture acid of PW and celite $(2.13 \mu \mathrm{g} / \mathrm{kg})(p<0.05)$. $\mathrm{BaP}$ reductions in sesame oil filtrated with a mixture of PW and other adsorbents such as kaolin and celite were smaller than that filtrated with only PW. On the other hand, $\mathrm{BaP}$ level in sesame oil filtrated with a mixture of acid clay and PW was reduced more considerably than those treated with PW-kaolin and PW-celite mixtures

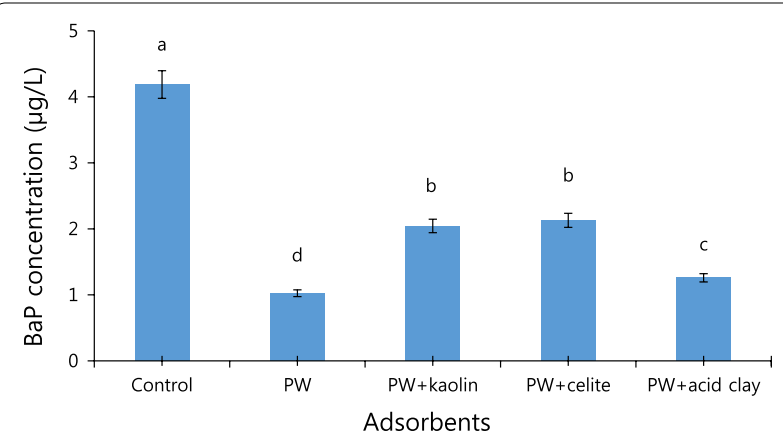

Fig. 2 Effects of the combination of activated carbon and other adsorbents on the reduction of benzo[a]pyrene in sesame oil. The symbols of each samples are as follows: Control, sesame oil sample with no treatment; $\mathrm{PW}$, sesame oil samples filtrated with powdered activated carbon made from wood; PW + kaolin, sesame oil samples filtrated with a mixture of PW and kaolin at a ratio 1:1; PW + celite, sesame oil samples filtrated with a mixture of PW and celite at a ratio 1:1; PW + acid clay, sesame oil samples filtrated with a mixture of PW and acid clay at a ratio 1:1. Data are expressed as mean $\pm \operatorname{SEM}(n=3)$. The presence of different letters $(a-d)$ indicates a statistical difference at $\mathrm{P}<0.05$ between bars $(p<0.05)$. Choi et al. [10] investigated how various adsorbents such as activated carbon, diatomaceous earth, kaolin, perlite, silicate, celite, and acid clay affected the reduction of $\mathrm{BaP}$ in sesame oil. They reported that activated carbon was the most effective adsorbent for reducing $\mathrm{BaP}$, which was consistent with the results of the present study. This might be explained by the ability of nonpolar adsorbents with low oxygen contents to efficiently adsorb PAHs such as BaP [17]. In addition, there was little difference between polar adsorbents such as celite, kaolin, and acid clay, possibly due to their relatively polar structure. Previous studies indicated that celite has a silica surface mostly composed of $\mathrm{SiO}_{2}(72 \%)$, and is covered by hydroxyl groups and oxygen bridges [18, 19]. In addition, clays such as kaolin and acid clay have the capability to adsorb positively charged compounds because the clay structures have a net negative charge [11]; however, they are used to remove PAHs due to their low cost.

\section{Effects of activated carbons on changes in volatile compounds}

Table 1 lists the effects of different adsorbents such as activated carbons on changes in the volatile profiles of sesame oil. In the sesame oil filtrated with absorbents, 61 volatile compounds including 18 pyrazines, 12 furan and furfurals, 13 thiazoles, 2 pyridines, 5 pyrroles, and 11 sulfur-containing compounds were identified.

Among the various volatile compounds, pyrazines, which are mostly produced by the Maillard reaction that occurs during thermal food processing [2], were predominant in the sesame oil samples in the present study. Pyrazines are major volatile compounds that contribute to the roasted and nutty odor notes in food products processed at high temperatures [20]. Some studies have found that levels of monoalkylpyrazines in deep-roasted sesame oil were relatively decreased, while there were more di- and trialkylpyrazines in deep-roasted sesame oil than in light-roasted sesame oil [5, 21, 22]. In particular, 2-ethyl-6-methylpyrazine, 2-ethyl-5-methylpyrazine, and 3-ethyl-2,5-dimethylpyrazine were important contributors to the flavor characteristics of sesame oil [15]. The present study found no significant differences in pyrazine content in the sesame oil samples filtrated with five activated carbons $(p>0.05)$. However, pyrazines such as 2-methylpyrazine, 2-ethenylpyrazine, 2-ethyl-3-methylpyrazine, 2-ethenyl-6-methylpyrazine, 2-acetylpyrazine, and 2-acetyl-3-methylpyrazine were significantly reduced when sesame oil was filtrated with acid clay combined with PW $(p<0.05)$. This might be explained by the relatively polar surface structure of acid clay and its negative net charge [11]. 


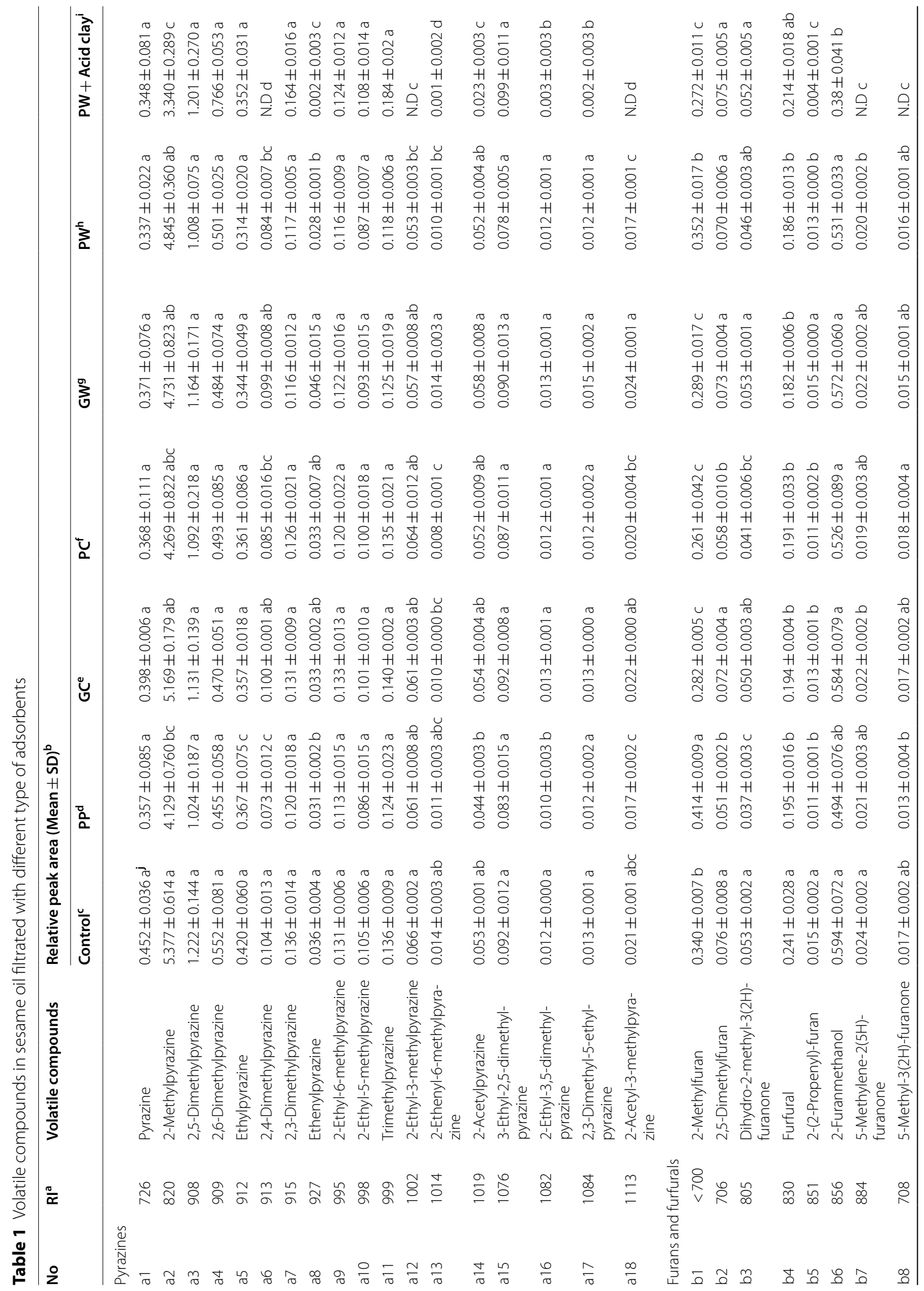




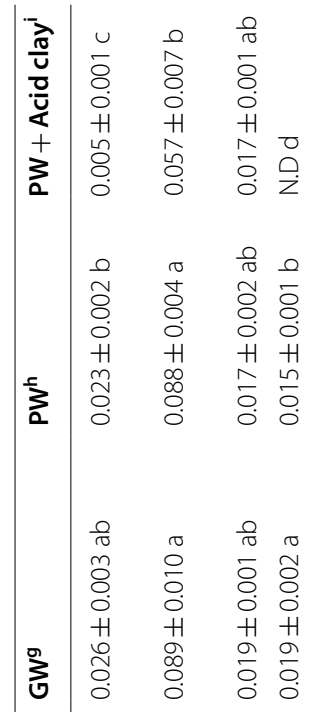

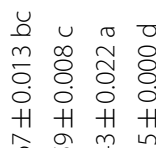

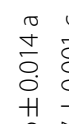

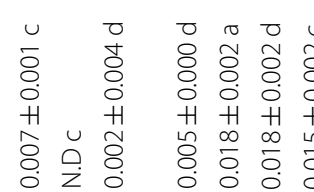

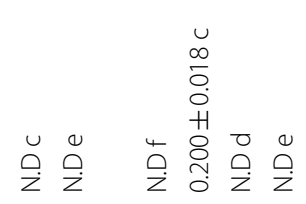

ช

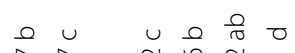

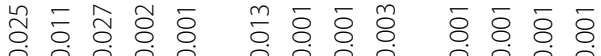

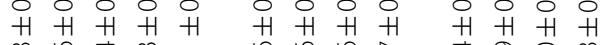

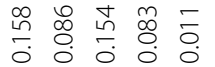

0 ช 6 0

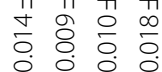

$\bar{\delta} \hat{8}$

H + H

H H H H

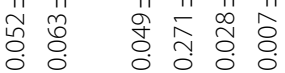

을

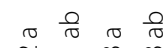

บ $\cup$ อ

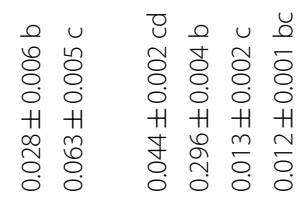

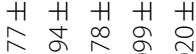

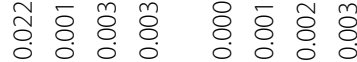

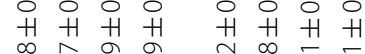

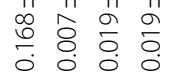

స.

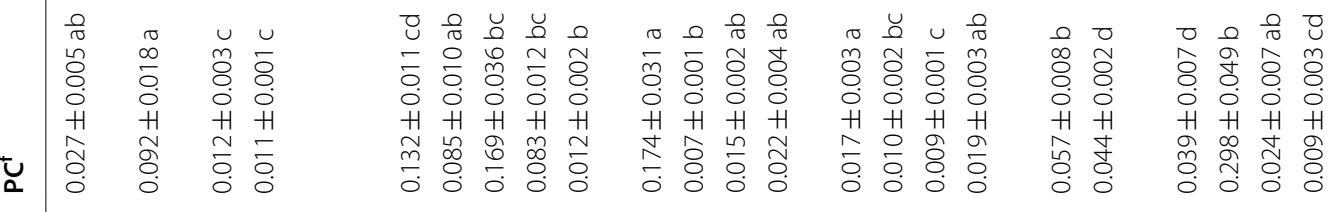

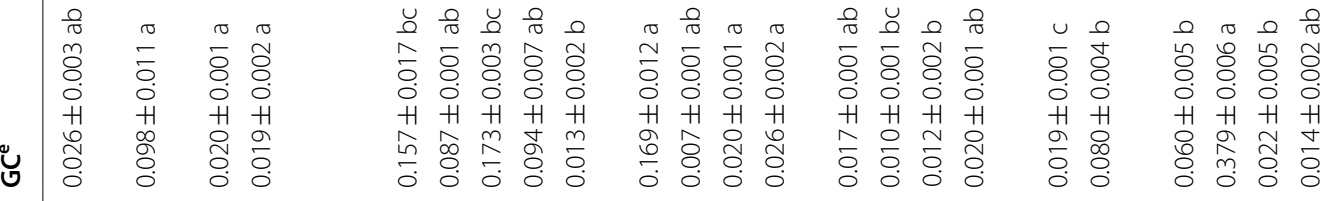

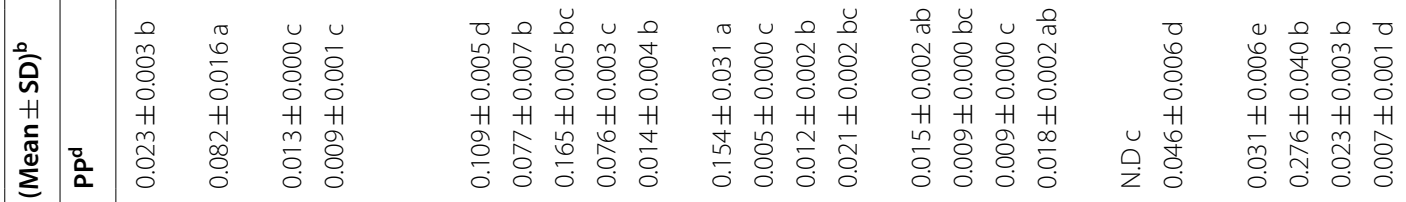

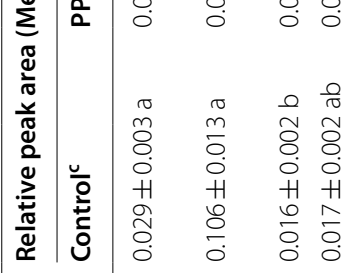

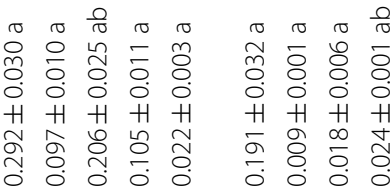

드늘

$\begin{array}{ll}0 & 0 \\ \circ & 8 \\ 0 & 8 \\ 0 & 0\end{array}$

$\begin{array}{llll}0 & 0 & a & 0\end{array}$

\begin{tabular}{llll}
0 & 8 & 8 & 8 \\
0 & 0 & 0 & 0 \\
\hline & 1 & 1
\end{tabular}

H H H H

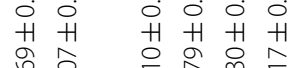

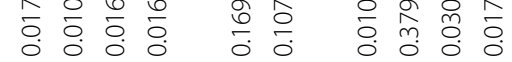

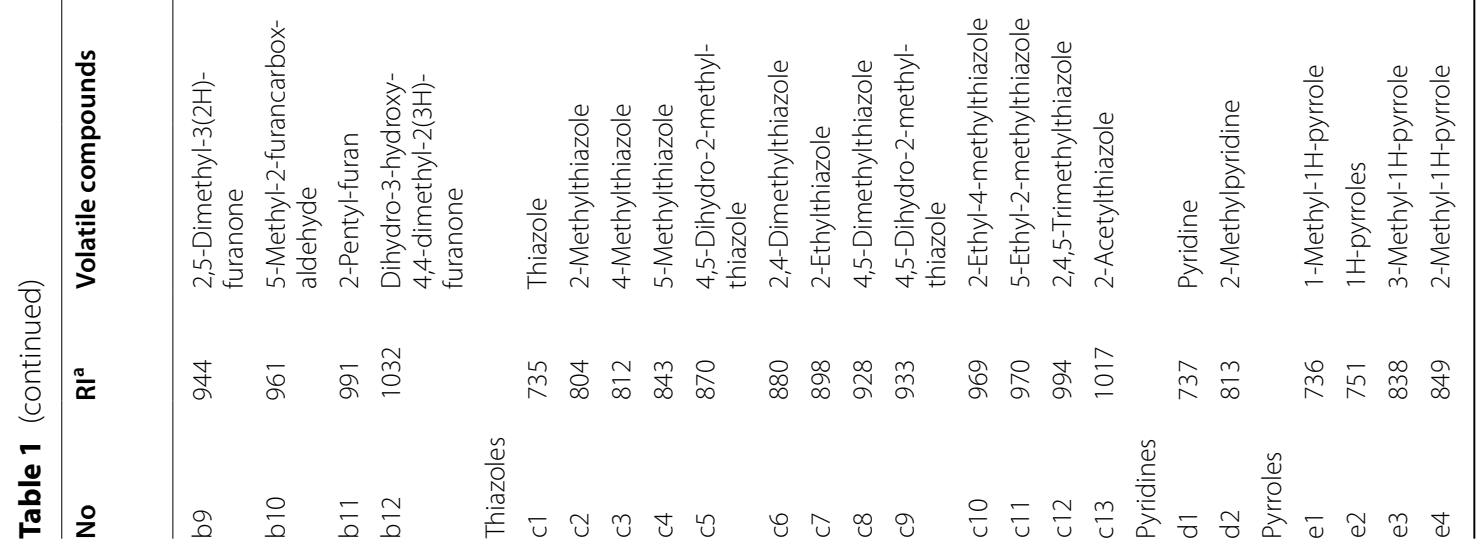




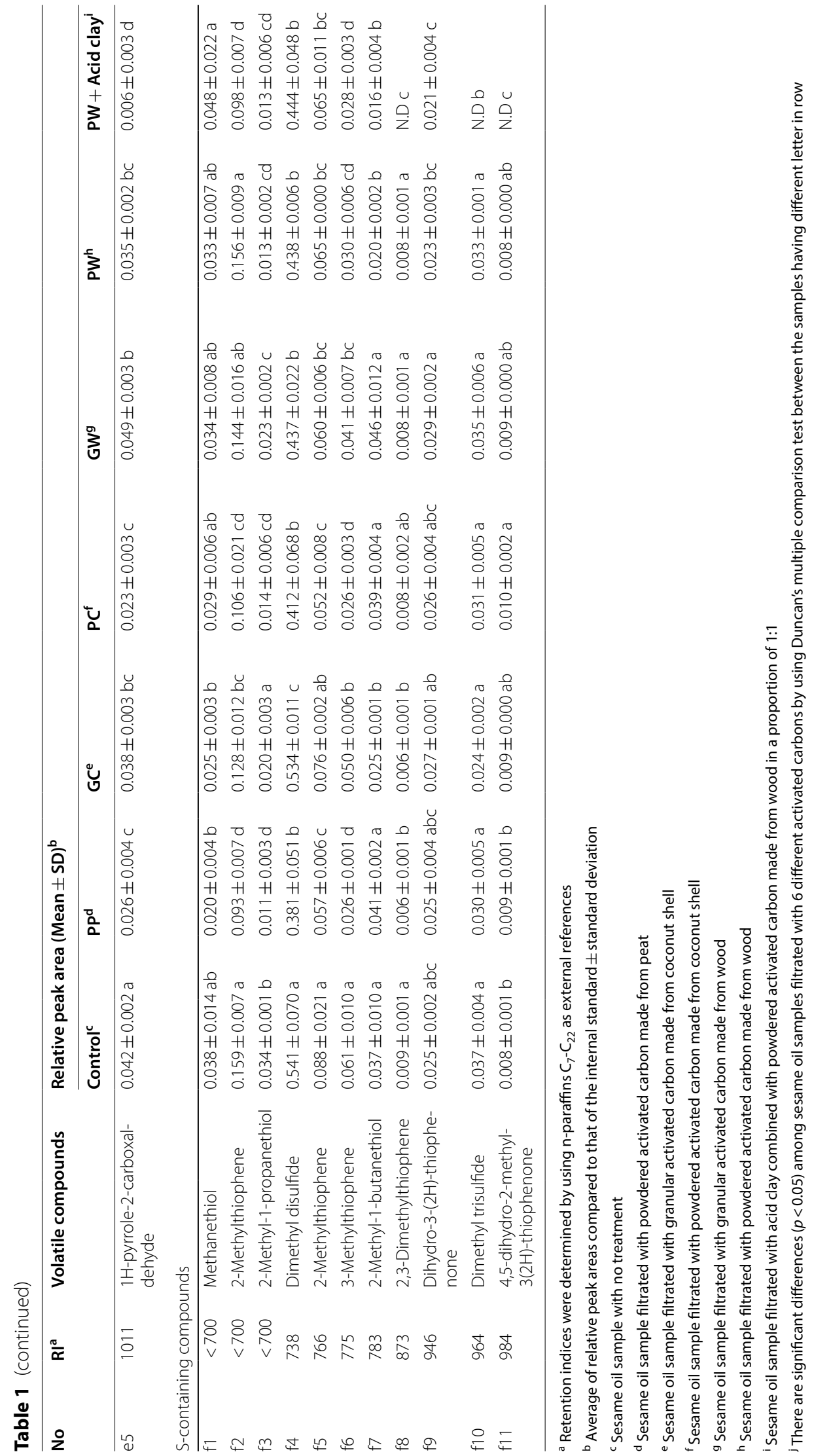


Furans are also considered major flavor constituents of sesame oil, with caramel-like and roasted odor notes [23]. Furans, including alkylfurans, which can be produced at high-temperature food processing, are mostly generated by carbohydrate and lipid degradation [24]. Some furans, such as 5-methylene- $2(5 \mathrm{H})$-furanone, 5-methyl-3(2H)-furanone, 2,5-dimethyl-3- $(2 \mathrm{H})$-furanone, 5-methyl-2-furancarboxaldehyde, and dihydro-3-hydroxy-4,4-dimethyl-2(3H)-furanone, were significantly reduced after filtration with acid clay combined with PW. This could be due to the relatively polar structure of furan compounds, especially furanones, which have more than one oxygen molecule, and the relatively polar structure of acid clay [11,25].

Thiazoles are major contributors to the pleasant sulfur and nutty odor notes in roasted sesame seeds [13]. Some thiazoles, such as thiazole, 4-methylthiazole, 5-methylthiazole, 4,5-dihydro-2-methylthiazole, 2-ethylthiazole, 4,5-dimethylthiazole, and 2,4,5-trimethylthiazole, detected in the present study were considerably reduced after filtration with powdered activated carbons. It might be due to the relatively large internal surface areas of the pores in activated carbons compared with those of granular activated carbons derived from coconut shells.

Pyrroles were important contributors to the burnt and earthy characteristic odor notes in sesame oil [15]. The amounts of pyrroles were greatly decreased in sesame oil samples filtrated with powdered activated carbon (PP, PC, and PW), while these compounds were slightly reduced in sesame oil samples filtrated with granular activated carbon (GC and GW). Especially, 1H-pyrrole2-carboxaldehyde, which has sweet and medicinal odor notes, was greatly decreased after filtration with powdered activated carbons (PP, PC, and PW).

Pyridines were detected in large amounts in sesame oil samples. The amounts of pyridines, including pyridine and 2-methylpyridine, were greatly decreased by filtration with powdered activated carbons due to the large internal surface area of the pores in these carbons.

Sulfur-containing compounds were also considerably decreased by filtration with activated carbons (PP, PC, PW, GC, and GW) in this study. Among sulfur-containing compounds, thiol compounds have predominantly roasted, green, nutty, and vegetable-like odor notes in sesame seed oil [15]. Increased thiol concentration could also be associated with the undesirable flavor of sesame oil as it induces a burnt or rubbery aroma from the formation of furfurylthiol [22]. In the present study, the concentration of sulfur-containing compounds such as thiols was also greatly decreased after filtration with powdered activated carbons (PP, PC, and PW) compared with filtration with granular activated carbons (GC and GW).
Overall, volatile compounds changed considerably when filtrated with powdered activated carbons (PP, PC, and PW) compared with granular activated carbons (GC and $\mathrm{GW})$. It could be explained that powdered activated carbons (PP, PC, and PW) showed higher adsorption efficiency of volatile compounds in sesame oil, possibly due to the large surface area of the internal pores in powdered activated carbons [26]. In particular, volatile compounds were greatly reduced after filtration with acid clay combined with PW at a 1:1 ratio. This might be due to the relatively ionic surface structure of acid clay [11]. Some studies explained the ionic characteristics of clays used in this study, demonstrating that clays have cation exchange capacity in addition to high specific surface area [27, 28]. In particular, their ionic characteristics could affect the adsorption and reduction of some volatiles, such as pyrazines, with positive ions depending the $\mathrm{pH}$. However, it would be more critical in aqueous systems rather than sesame oil.

\section{Acknowledgements}

This work was supported by a Grant (13162MFDS051-2) from the Ministry of Food and Drug Safety in 2014 and BK21 FOUR (Fostering Outstanding Universities for Research, 4299990914600).

\section{Authors' contributions}

Conceptualization, Y-SK; Experiments, J-YJ; Analysis, SML and J-GL; Investigation, Y-SK and J-GL; Data Curation, J-YJ and SML; Writing-Original Draft Preparation, SML and J-YJ; Writing-Review and Editing, Y-SK and SML; Supervision, Y-SK. All authors read and approved the final manuscript.

\section{Declarations}

\section{Competing interests}

The authors declare that they have no competing interests.

\section{Author details}

${ }^{1}$ Department of Food and Nutrition, Inha University, Incheon 22212, Republic of Korea. ${ }^{2}$ Department of Food Science and Engineering, Ewha Womans University, Seoul 03760, Republic of Korea. ${ }^{3}$ Department of Food Biotechnology, Dong-A University, Busan 49315, Republic of Korea.

Received: 29 September 2021 Accepted: 22 November 2021

Published online: 28 November 2021

\section{References}

1. Abou-Gharbia HA, Shehata AAY, Shahidi F (2000) Effect of processing on oxidative stability and lipid classes of sesame oil. Food Res Int 33(5):331-340

2. Manley C, Vallon P, Erickson R (1974) Some aroma components of roasted sesame seed (sesamum indicum L.). J Food Sci 39(1):73-76

3. Lee SH, Joo KJ (1998) Analysis of volatile flavor compounds in sesame oil extracted by purge-and-trap method. Korean J Food Sci Technol 30(2):260-265

4. Yen GC (1990) Influence of seed roasting process on the changes in composition and quality of sesame (Sesame indicum) oil. J Sci Food Agric 50(4):563-570

5. Kim H, Choi C, Woo S (1998) Changes of volatile flavor compounds in sesame oils during industrial process. Korean J Food Sci Technol 30(4):739-744 
6. Yoshida H (1994) Composition and quality characteristics of sesame seed (sesamum indicum) oil roasted at different temperatures in an electric oven. J Sci Food Agric 65(3):331-336

7. João Ramalhosa M, Paíga P, Morais S, Delerue-Matos C, Prior Pinto Oliveira MB (2009) Analysis of polycyclic aromatic hydrocarbons in fish: Evaluation of a quick, easy, cheap, effective, rugged, and safe extraction method. J Sep Sci 32(20):3529-3538

8. Seo I, Nam H, Shin H (2009) Influence of polycyclic aromatic hydrocarbons formation in sesame oils with different roasting conditions. Korean J Food Sci Technol 41(4):355-361

9. Haritash A, Kaushik C (2009) Biodegradation aspects of polycyclic aromatic hydrocarbons (PAHs): A review. J Hazard Mater 169(1):1-15

10. Choi SK, Choe SB, Kang ST (2014) Reduction of benzo(a)pyrene content in sesame oil by using adsorbents. J Korean Soc Food Sci Nutr 43(4):564-569

11. Crini G (2006) Non-conventional low-cost adsorbents for dye removal: A review. Bioresour Technol 97(9):1061-1085

12. Yang $X, A l-D u r i B(2005)$ Kinetic modeling of liquid-phase adsorption of reactive dyes on activated carbon. J Colloid Interface Sci 287(1):25-34

13. Nakamura S, Nishimura O, Masuda H, Mihara S (1989) Identification of volatile flavor components of the oil from roasted sesame seeds. Agr Biol Chem 53(7):1891-1899

14. Park D, Maga JA, Johnson DL, Morini G (1995) Major volatiles in toasted sesame seed oil. J Food Lipids 2(4):259-268

15. Shimoda M, Shiratsuchi H, Nakada Y, Wu Y, Osajima Y (1996) Identification and sensory characterization of volatile flavor compounds in sesame seed oil. J Agric Food Chem 44(12):3909-3912

16. Lee D, Noh B, Bae S, Kim K (1998) Characterization of fatty acids composition in vegetable oils by gas chromatography and chemometrics. Anal Chim Acta 358(2):163-175

17. Valderrama C, Cortina J, Farran A, Gamisans X, Lao C (2007) Kinetics of sorption of polyaromatic hydrocarbons onto granular activated carbon and macronet hyper-cross-linked polymers (MN200). J Colloid Interface Sci 310(1):35-46

18. Al-Ghouti M, Khraisheh M, Allen S, Ahmad M (2003) The removal of dyes from textile wastewater: A study of the physical characteristics and adsorption mechanisms of diatomaceous earth. J Environ Manage 69(3):229-238

19. Khraisheh M, Al-Ghouti M, Allen S, Ahmad M (2005) Effect of $\mathrm{OH}$ and silanol groups in the removal of dyes from aqueous solution using diatomite. Water Res 39(5):922-932

20. Coleman EC, Ho C (1980) Chemistry of baked potato flavor. 1. pyrazines and thiazoles indentified in the volatile flavor of baked potato. J Agric Food Chem 28(1):66-68

21. Park MH, Jeong MK, Yeo J, Son H, Lim C, Hong EJ (2011) Application of solid phase-microextraction (SPME) and electronic nose techniques to differentiate volatiles of sesame oils prepared with diverse roasting conditions. J Food Sci 76(1):C80-C88

22. Schieberle $P$ (1996) Odour-active compounds in moderately roasted sesame. Food Chem 55(2):145-152

23. Rey-Salgueiro L, García-Falcón MS, Martínez-Carballo E, Simal-Gándara J (2008) Effects of toasting procedures on the levels of polycyclic aromatic hydrocarbons in toasted bread. Food Chem 108(2):607-615

24. Maga JA, Katz I (1979) Furans in foods. Crit Rev Food Sci Nutr 11(4):355-400

25. Chen H, Hodges LM, Liu R, Stevens WCJ, Sabat M, Harman WD (1994) Dearomatization of furan: Structure of an. eta. 2-furan complex and a survey of its reactivity. J Am Chem Soc 116(12):5499-5500

26. Newcombe G, Drikas M, Hayes R (1997) Influence of characterised natural organic material on activated carbon adsorption: II. Effect on pore volume distribution and adsorption of 2-methylisoborneol. Water Res 31(5):1065-1073

27. Bibi I, Singh B, Silvester E (2014) Dissolution kinetics of soil clays in sulfuric acid solutions: Ionic strength and temperature effects. Appl Geochem 51:170-183

28. Bhattacharyya KG, Gupta SS (2011) Removal of Cu (II) by natural and acid-activated clays: An insight of adsorption isotherm, kinetic and thermodynamics. Desalination 272(1-3):66-75

\section{Publisher's Note}

Springer Nature remains neutral with regard to jurisdictional claims in published maps and institutional affiliations.

\section{Submit your manuscript to a SpringerOpen ${ }^{\circ}$ journal and benefit from:}

- Convenient online submission

- Rigorous peer review

- Open access: articles freely available online

- High visibility within the field

- Retaining the copyright to your article

Submit your next manuscript at $\mathbf{s p r i n g e r o p e n . c o m ~}$ 